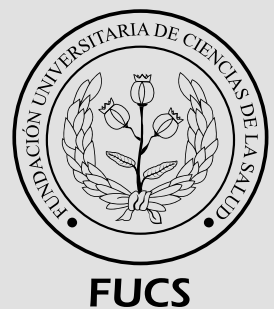

\title{
Análisis de datos en el estudio del COVID-19 en Colombia
}

Jorge Enrique Díaz-Pinzón ${ }^{\text {a }}$

\section{Analysis of data in the COVID-19 study in Colombia}

a Ingeniero. Magister en Gestión de la Tecnología Educativa, Especialista en Administración de la Informática Educativa, Docente de matemáticas e Investigador, Secretaría de Educación de Soacha, Cundinamarca.

\section{R E S U M E N}

Introducción: a finales de 2019 la aparición de SARS-CoV-2 que causa el COVID-19, desató una pandemia que se ha asociado con más de 500.000 muertes en todo el mundo hasta julio 2020, siendo el brote original en la provincia de Hubei, China el cual se ha extendido a otros países. Objetivo: analizar los datos de la información en Colombia para COVID-19. Metodología: para desarrollar la investigación se utilizó la base de datos de las personas contagiadas, recuperadas, y fallecidas por el COVID-19. La información de los datos corresponde al período acumulado del 6 de marzo al 21 de septiembre de 2020 para Colombia. Resultados: se estimó a 21 de septiembre 2020, las medidas de tendencia central, la primera, la media, para el caso de los contagios fue de $185.757,25$, para recuperados $122.771,21$ y para fallecidos de $6.602,60$. Para la mediana se ubica en $49.842,50$ para contagiados, 19.624 para recuperados, y 1.629,5 para fallecidos, y para el caso de la moda, 1 para los contagiados, 0 para recuperados y fallecidos. El mínimo de personas contagiadas fue de 1, con un máximo de 770.435, para el caso de recuperados

\section{INFORMACIÓN DEL ARTÍCULO}

Historia del artículo: Fecha recibido: septiembre 30 de 2020 Fecha aceptado: octubre 5 de 2020
Autor para correspondencia. Ing. Jorge Enrique Díaz Pinzón jediazp@unal.edu.co 
el mínimo fue de 0, y un máximo de 640.900, y para los fallecidos, un mínimo de 0 y un máximo de 24.397. Conclusiones: la incidencia y prevalencia de la enfermedad probablemente seguirá aumentando hasta que no se encuentre una solución definitiva a la pandemia propiciada por el COVID-19. Es bien significativo estudiar la dinámica de posibles brotes infecciosos en nuestro país, ya que esta enfermedad recién surgida ha tenido un rápido crecimiento contagioso.

Palabras clave: COVID-19, pandemia, análisis de datos.

(C) 2020 Fundación Universitaria de Ciencias de la Salud - FUCS. Este es un artículo Open Access bajo la licencia CC BY-NC-ND (http://creativecommons.org/licenses/by-nc-nd/4.0/).

\section{ABSTRACT}

Introduction: in late 2019, the newly emerged SARS-CoV-2, which causes COVID-19, triggered a pandemic that has been associated with more than 500.000 deaths worldwide, as of July 2020. The source of the outbreak was the province of Hubei, China, spreading to other countries. Objective: to analyze the COVID-19 data in Colombia. Methodology: The database including people infected with COVID-19, the individuals who have recovered and those who died from COVID-19 was used for the research, with the information accumulated in Colombia between March 6 and September 21 2020. Results: the measures of central location were estimated as of September 21 2020. The mean for infected cases was 185.757,25, 122.771,21 for recovered individuals and 6.602,60 for COVID-19-related deaths. The median for infected cases was 49.842,50, 19.624 for recoveries and 1.629,5 for deaths. The mode was 1 for those infected and 0 for those who recovered or died. The minimum of people infected was 1 , and the maximum was 770.435 . For individuals who recovered the minimum was 0 , and the maximum was 640.900. And for those who died, the minimum was 0 and the maximum was 24.397. Conclusions: the incidence and prevalence of the disease will probably continue to rise until a definitive solution to the pandemic caused by COVID-19 is found. It is crucial to study the dynamics of possible infectious disease outbreaks in our country, since this newly emerged disease has shown a rapid contagion rate.

Key words: COVID-19, pandemic, data analysis

(C) 2020 Fundación Universitaria de Ciencias de la Salud - FUCS. This is an open access article under the CC BY-NC-ND license (http://creativecommons.org/licenses/by-nc-nd/4.0/).

\section{INTRODUCCIÓN}

A finales de 2019 la aparición de SARS-CoV-2 que causa COVID-19 desató una pandemia que se ha asociado con más de 500.000 muertes en todo el mundo hasta julio de 2020. El brote original inició en la provincia de Hubei, China y se extendió a otros países. ${ }^{1,2,3}$

A medida que disminuyen las tasas de casos nuevos, los países están implementando varias estrategias de salida para relajar las restricciones. ${ }^{4}$ El éxito a largo plazo de cualquier estrategia de salida potencial depende de lo que suceda a nivel regional, ya que la importación internacional podría abrumar los esfuerzos para evitar el resurgimiento mediante pruebas y rastreo de contactos. ${ }^{5}$

La experiencia en los países hasta la fecha ha enfatizado la intensa presión que una epidemia de COVID-19 ejerce sobre los sistemas nacionales de salud, con la demanda de camas de cuidados intensivos y ventiladores mecánicos que superan rápidamente su disponibilidad, incluso en entornos de recursos relativamente altos. ${ }^{6}$ Esto tiene consecuencias potencialmente profundas para los entornos de escasos recursos, donde la calidad y disponibilidad de la atención médica y los recursos relacionados (como el oxígeno) suelen ser deficientes. ${ }^{7}$ Actualmente se viene desarrollando una vacuna para prevenir la enfermedad por coronavirus 2019 (COVID-19) es un imperativo mundial, y definir lo que está en juego y los posibles obstáculos es fundamental porque las decisiones regulatorias y médicas se basan en cálculos de beneficio: riesgo. ${ }^{8}$

Actualmente cualquier estudio epidemiológico de cierta rigidez, maneja para el análisis de datos paquetes estadísticos, como el EPIINFO y el SPSS, no por ello convenimos de pasar de largo sobre los conceptos estadísticos y epidemiológicos, en los que estos programas se apoyan. ${ }^{9}$

En el presente trabajo de investigación se analizarán los datos del COVID-19 en Colombia, desde el registro del primer contagiado el 6 de marzo de 2020, hasta el 21 de septiembre de 2020, correspondiente a 200 días de información, la cual se obtuvo de la página del Instituto Nacional de Salud. ${ }^{10}$ 


\section{RESULTADOS}

Con la ayuda del paquete estadístico SPSS v.25, se construyeron las tablas 1 y 2. En la tabla 1 se aprecian los datos estadísticos acumulados de las personas contagiadas, recuperadas y fallecidas, en el período comprendido entre el 6 de marzo al 21 de septiembre de 2020, es decir un total de 200 días de observación. En la tabla se aprecia las medidas de tendencia central, la primera, la media, para el caso de los contagios fue de 185.757,25, para recuperados 122.771,21, y para fallecidos de $6.602,60$. Para la mediana se ubica en 49.842,50 para contagiados, 19.624 para recuperados, y $1.629,5$ para fallecidos, y para el caso de la moda, 1 para los contagiados, 0 para recuperados y fallecidos.

El mínimo de personas contagiadas fue de 1, con un máximo de 770.435, para el caso de recuperados el mínimo fue de 0 , y un máximo de 640.900, y para los fallecidos, un mínimo de 0 y un máximo de 24.397.

Tabla 1. Estadística de datos acumulados

\begin{tabular}{|l|c|c|c|}
\multicolumn{1}{c|}{} & Contagiados & Recuperados & Fallecidos \\
\hline N Válido & 200 & 200 & 200 \\
\hline \multicolumn{1}{|c|}{ Perdidos } & 0 & 0 & 0 \\
\hline Media & 185757,25 & 122771,21 & 6062,60 \\
\hline Mediana & $49842,50^{\mathrm{a}}$ & $19624,00^{\mathrm{a}}$ & $1629,50^{\mathrm{a}}$ \\
\hline Moda & 1 & 0 & 0 \\
\hline Varianza & 58770946106,862 & 35507723845,269 & 60109143,689 \\
\hline Rango & 770434 & 640900 & 24397 \\
Mínimo & 1 & 0 & 0 \\
\hline Máximo & 770435 & 640900 & 24397 \\
\hline
\end{tabular}

Se ha calculado a partir de datos agrupados.

Fuente. Elaboración propia.
En la tabla 2 se observan los datos estadísticos a diario de las personas contagiadas, recuperadas y fallecidas, en el período comprendido entre el 6 de marzo al 21 de septiembre de 2020, es decir un total de 200 días de observación. En la tabla se aprecia las medidas de tendencia central, la primera, la media, para el caso de los contagios fue de 3.852,18, para recuperados 3.204,5, y para fallecidos de 121,98. Para la mediana se ubica en 1.878 para contagiados, $1.006,5$ para recuperados, y 62,0 para fallecidos, y para el caso de la moda, 0 para los contagiados, recuperados y fallecidos.

El mínimo fue de 0 para personas contagiadas, recuperadas y fallecidas, y un máximo de 13.056 para personas contagiadas, para el caso de recuperados 16.594, y un máximo de 400 para los fallecidos.

Tabla 2. Estadística de datos a diario

Contagiados-Día Recuperados-Día Fallecidos-Día

\begin{tabular}{|l|c|c|c|}
\hline N Válido & 200 & 200 & 200 \\
\hline \multicolumn{1}{|c|}{ Perdidos } & 0 & 0 & 0 \\
Media & 3852,18 & 3204,50 & 121,98 \\
Mediana & $1878,00^{\mathrm{a}}$ & $1006,50^{\mathrm{a}}$ & $62,00^{\mathrm{a}}$ \\
Moda & 0 & 0 & 0 \\
\hline Varianza & 15937993,854 & 17817796,623 & 15120,020 \\
Rango & 13056 & 16594 & 400 \\
Mínimo & 0 & 0 & 0 \\
\hline Máximo & 13056 & 16594 & 400 \\
Suma & 770435 & 640900 & 24396 \\
\hline
\end{tabular}

Se ha calculado a partir de datos agrupados.

Fuente. Elaboración propia.

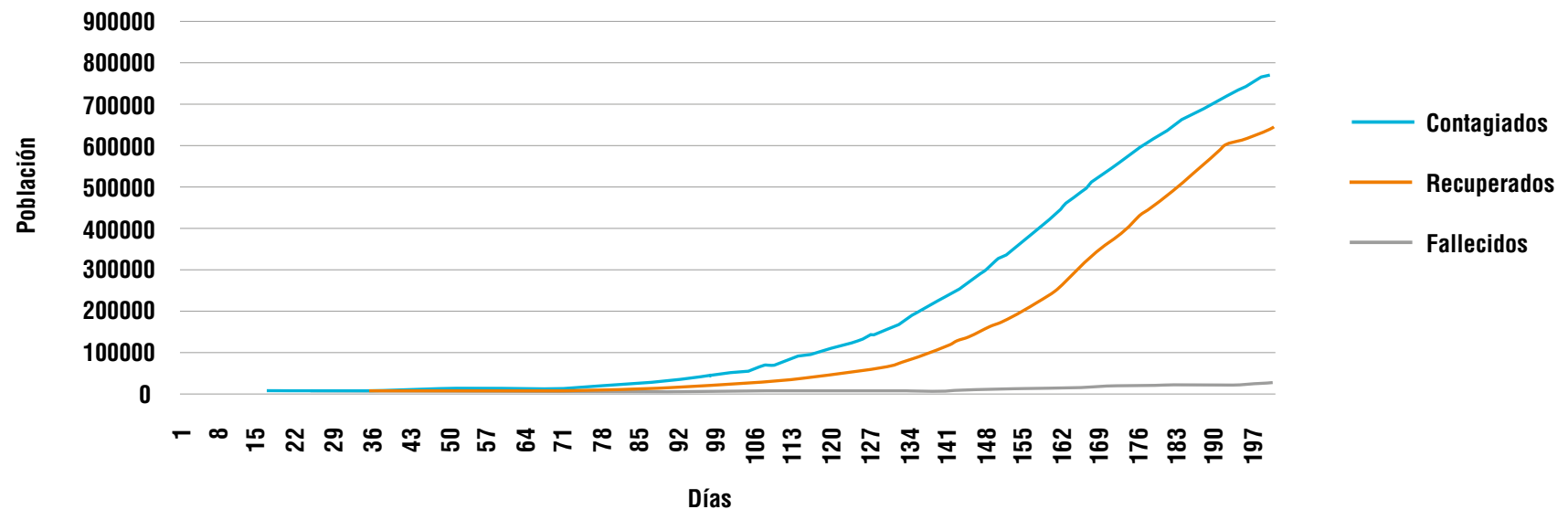

Figura 1. Tendencia acumulada para contagios, recuperados y fallecidos por COVID-19. Fuente. Elaboración propia. 


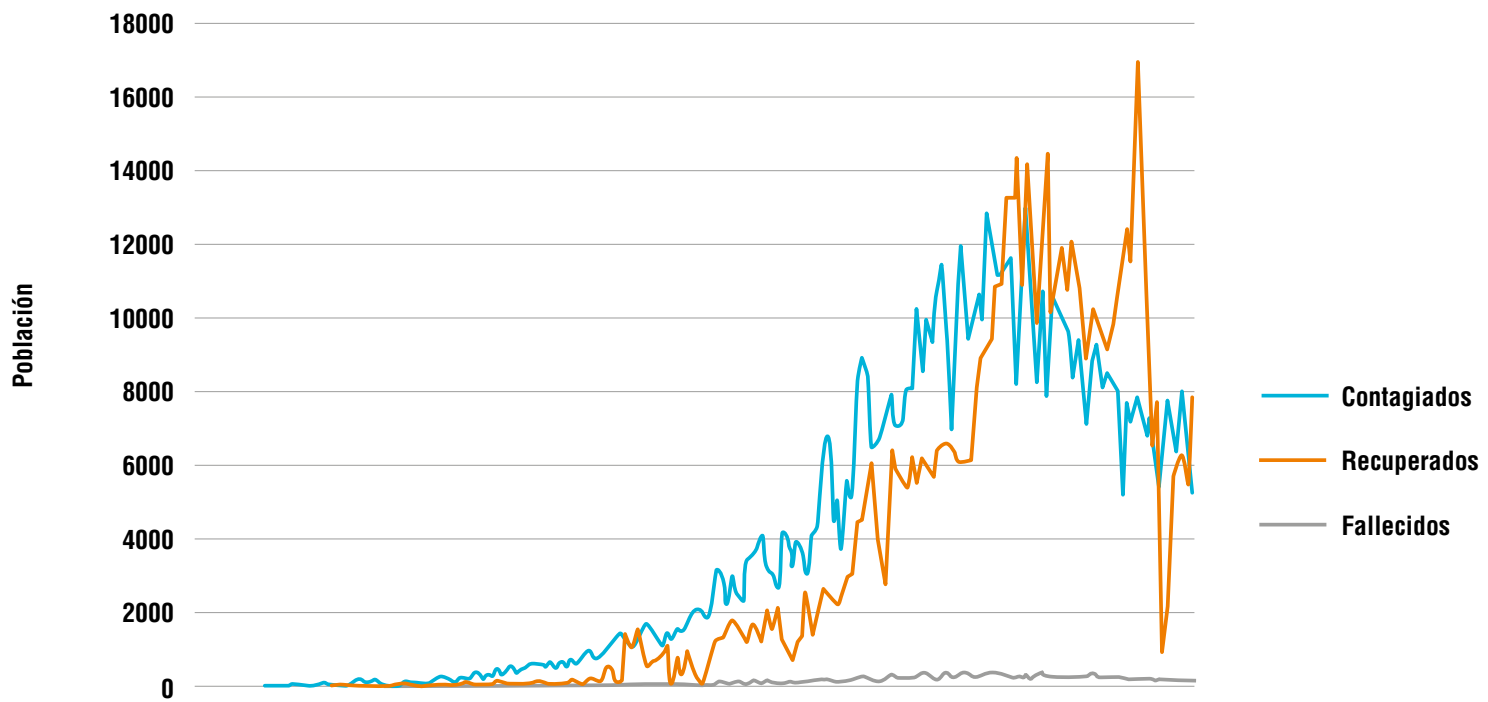

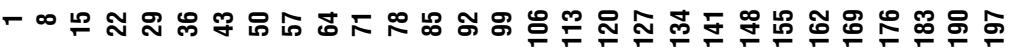

Días

Figura 2. Tendencia diaria para contagiados, recuperados, y fallecidos por COVID-19. Fuente. Elaboración propia.

En la figura 1 se aprecian las tendencias de personas contagiadas, recuperadas, y fallecidas, durante el período 6 de marzo a 21 de septiembre de 2020, para los contagiados y recuperados la tendencia es exponencial, y para los fallecidos lineal.

En la figura 2 se observan las tendencias de personas contagiadas, recuperadas, y fallecidas a diario, se puede establecer que para el 19 de agosto de 2020 (día 167), se presentó el mayor pico de contagio con 13.056, para el día 10 de septiembre de 2020 (día 189), se ubicó el pico mayor de personas recuperadas con 16.594, y para el día 22 de agosto de 2020 (día 170), se apreció el mayor pico de fallecimientos con 400 .

\section{CONCLUSIÓN}

Se estimó a 21 de septiembre de 2020 que las medidas de tendencia central, la primera, la media, para el caso de los contagiados fue de 185.757,25, para recuperados $122.771,21$, y para fallecidos de $6.602,60$. Para la mediana se ubica en 49.842,50 para contagiados, 19.624 para recuperados y $1.629,5$ para fallecidos, y para el caso de la moda, 1 para los contagiados, 0 para recuperados y fallecidos. El mínimo de personas contagiadas fue de 1 , con un máximo de 770.435 , para el caso de recuperados el mínimo fue de 0 , y un máximo de 640.900, y para los fallecidos, un mínimo de 0 y un máximo de 24.397.

Para los casos a diario, se estimó que las medidas de tendencia central, la primera, la media, para el caso de los contagios fue de 3.852,18, para recuperados 3.204,5, y para fallecidos de 121,98 . Para la mediana se ubica en 1.878 para contagiados, $1.006,5$ para recuperados, y 62,0 para fallecidos, y para el caso de la moda, 0 para los contagiados, recuperados y fallecidos.

El mínimo fue de 0 para personas contagiadas, recuperadas y fallecidas, y un máximo de 13.056 para personas contagiadas, para el caso de recuperados 16.594, y un máximo de 400 para los fallecidos.

La incidencia y prevalencia de la enfermedad, probablemente seguirá aumentando hasta que no se encuentre una solución definitiva a la pandemia propiciada por el COVID-19. ${ }^{11}$ Es significativo estudiar la dinámica de posibles brotes infecciosos del COVID-19 en nuestro país ya que esta enfermedad recién surgida ha tenido un rápido crecimiento de contagio. ${ }^{12}$

\section{REFERENCIAS}

1. Mbow M, Lell B, Jochems SP, Cisse B, Mboup S, Dewals BG, et al. COVID-19 in Africa: Dampening the storm?. Science. 2020;369(6504):624-6. doi: 10.1126/science.abd3902

2. Worobey M, Pekar J, Larsen BB, Nelson MI, Hill V, Joy JB, et al. The emergence of SARS-CoV-2 in Europe and North America. Science. 2020. doi: 10.1101/2020.05.21.109322

3. Maier BF, Brockmann D. Effective containment explains subexponential growth in recent confirmed COVID-19 cases in China. Science. 2020;368(6492):742-6. doi: 10.1126/science. abb4557 
4. Colbourn T. COVID-19: extending or relaxing distancing control measures. The Lancet Public health. 2020;5(5):e236-e7. doi: 10.1016/S2468-2667(20)30072-4

5. Lai S, Bogoch I, Ruktanonchai N, Watts A, Lu X, Yang W, et al. Assessing spread risk of Wuhan novel coronavirus within and beyond China, January-April 2020: a travel network-based modelling study. medRxiv. 2020. doi: 10.1101/2020.02.04.20020479

6. Remuzzi A, Remuzzi G. COVID-19 and Italy: what next? The Lancet. 2020;395(10231):1225-8. doi: 10.1016/S0140-6736(20)30627-9

7. Ginsburg AS, Van Cleve WC, Thompson MI, English M. Oxygen and pulse oximetry in childhood pneumonia: a survey of healthcare providers in resource-limited settings. Journal of tropical pediatrics. 2012;58(5):389-93. doi: 10.1093/tropej/fmr103

8. Graham BS. Rapid COVID-19 vaccine development. Science. 2020;368(6494):945-6. doi: 10.1126/science.abb8923
9. García Salinero J. Análisis de datos en los estudios epidemiológicos I. Nure Investigación,. 2005; 16:1-5.

10. Instituto Nacional de Salud. Coronavirus (CO-VID-19) en Colombia [Internet]. Colombia: Instituto Nacional de Salud; 2020 [cited 2020 agosto]; Available from: https://bit.ly/2UNnOtl.

11. Díaz Pinzón JE. Estimación de la Prevalencia del COVID-19 en Colombia. Repert Med Cir. 2020;29(Núm. Supl.1):99-102. doiI: 10.31260/REPERTMEDCIR.01217372.1115

12. Díaz Pinzón JE. Proyección del COVID-19 en Colombia. Rev Med. 2020;28(1):11-20. 